\title{
Retour vers le Médiopassif
}

\author{
Marie Laurence Knittel* \\ Université de Lorraine \& ATILF
}

\begin{abstract}
*Résumé. Nous proposons une analyse du médiopassif fondée sur sa comparaison avec d'autres structures à renversement, notamment les anticausatives, les formes passives et les constructions à sujet instrumental. Nous montrons que les structures médopassives sont caractérisées par 3 propriétés principales : le caractère événementiel de la forme transitive correspondante, la valeur strictement non-épisodique, et l'aspect imperfectif.
\end{abstract}

\begin{abstract}
Back to the Middle We analyze mediopassive structures in comparing them with others where the surface subjects differ from that of their transitive counterparts: anticausatives, passives and sentences with instrumental subjects. We show that mediopassives display 3 major properties: they alternate with eventive structures, exhibit a non-episodic value and are strictly imperfective.
\end{abstract}

\section{Introduction}

Le médiopassif, ou 'moyen' (Kemmer 1993), illustré par les exemples (1) ci-dessous, fait partie des voix non-actives du verbe, au même titre que le passif.

1. a. La cathédrale se voit de loin

b. La laine se lave en machine

c. La poubelle se sort chaque soir

A partir des travaux antérieurs de Obenauer (1970), Ruwet (1972), Lamiroy (1993), Lagae (2002) et Zribi-Hertz (1982, 2008) et des exemples qu'ils présentent, complétés par des exemples attestés, notre objectif est de proposer une analyse unifiée du phénomène. Notre hypothèse est que les structures médopassives sont caractérisées par 3 propriétés principales : le caractère événementiel de la forme transitive correspondante, la valeur nonépisodique - à une exception près —, et l'aspect imperfectif. Notre analyse se fonde sur la comparaison du médiopassif avec d'autres structures à renversement, notamment les anticausatives, les formes passives en être + Vé correspondantes et les constructions à sujet instrumental.

*marie-laurence.knittel@univ-lorraine.fr 


\section{Description}

\subsection{Structure argumentale}

Les phrases médiopassives sont des constructions réfléchies de verbes transitifs directs, dans lesquelles l'argument initialement complément du verbe occupe la position sujet en surface, comme cela est illustré dans la schématisation (2).

\section{2. a. SN1 V SN2 \\ b. $\quad \mathrm{SN} 2 \mathrm{se}-\mathrm{V}$}

Ruwet (1972) et Lamiroy (1993) considèrent que les phrases médiopassives se caractérisent par la présence implicite d'un agent humain arbitraire, qui peut être restitué par le pronom on dans les équivalents transitifs des médiopassifs :

3. a. Les feuilles mortes se ramassent à la pelle

$=\quad$ b. On ramasse les feuilles mortes à la pelle

4. a. Les prises de sang se font dans cette salle

$=\quad$ b. On fait les prises de sang dans cette salle

5. a. La choucroute se mange en hiver

$=\quad$ b. On mange la choucroute en hiver

Cette caractérisation semble indiquer que les phrases médiopassives sont des structures nécessairement dérivées de phrases agentives. Cependant, un exemple tel que (6), nous indique que cela n'est pas nécessairement le cas. En effet, comme le montre l'impossibilité d'utiliser l'adverbe volontairement, le sujet du verbe voir n'est pas analysable comme un Agent, mais plutôt comme un Experiencer.

6. a. La cathédrale se voit de loin

$=$ b. On voit $\left({ }^{*}\right.$ volontairement) la cathédrale de loin.

Toutefois, l'inacceptabilité des exemples (7-8) indique que les sujets animés nonhumains, même s'ils se caractérisent comme des initiateurs de l'action décrite par le verbe, sont difficilement acceptables :

7. a. Les chiens mordent les facteurs

b. ?? Les facteurs se mordent toujours

8. a. Les sauterelles ravagent les récoltes régulièrement

b. ?? Les récoltes se ravagent régulièrement

La caractérisation appropriée des verbes pouvant donner lieu à des structures médiopassives semble donc être le caractère humain plutôt qu'agentif de leurs sujets originels. Nous rejoignons ainsi l'observation de Reinhart \& Siloni (2005, in Zribi-Hertz 2008), qui caractérisent ces entités comme conscientes.

Ceci constitue donc une restriction lexicale sur le médiopassif.

\subsection{Valeur aspectuelle}

Obenauer (1970) et Ruwet (1972) notent que le médiopassif dispose d'une valeur aspectuelle particulière : il ne décrit pas des événements ancrés temporellement, ce dont témoignent les exemples ci-dessus. Ceci est confirmé par l'impossibilité d'employer le 
progressif être en train de qui, au présent, permet de décrire un événement en cours au moment d'énonciation.

9. a. ${ }^{*}$ Ce sport est en train de se pratiquer

b. *Les feuilles mortes sont en train de se ramasser

c. *Les prises de sang sont en train de se faire

Les phrases médiopassives n'ont donc pas en principe d'interprétation épisodique. Cependant, ce point de vue est contesté, et certains auteurs considèrent certaines structures réfléchies et non-agentives à valeur épisodique comme des médiopassifs. Ce point sera discuté dans la section 6 .

\subsection{Présence d'un constituant supplémentaire}

La seconde caractéristique du médiopassif repérée dans la littérature (Obenauer 1970, Zribi-Hertz 1982) est la présence d'un constituant supplémentaire de type circonstanciel. Ce constituant peut indiquer la manière (10), éventuellement sous la forme d'un prédicat adjectival portant sur le sujet (11), le lieu (12), le temps (13), ou renvoyer à un instrument (14).

10. a. Un rosier se taille à la main

b. Contrairement au rhume ou à la grippe, Ebola s'attrape difficilement ${ }^{\mathrm{i}}$

11. a. Les pommes de terres se mangent cuites

b. Ce bouillon se boit chaud ou froid ${ }^{\mathrm{ii}}$

12. a. Les prises de sang se font dans cette salle

b. L'anatomie s'enseigne en fac de médecine

13. a. Le ski se pratique en hiver

b. Les prises de sang se font le matin

14. a. Cette peinture s'étale au rouleau

b. Les œufs en neige se montent au batteur électrique

Toutefois, même si de tels circonstanciels sont fréquents, ils ne sont en rien obligatoires, comme l'indiquent la bonne formation des exemples (15).

15. a. Un rosier se taille

(i.e. un rosier doit être taillé)

b. Ces champignons (ne) se mangent (pas) (i.e. sont / ne sont pas comestibles)

\section{Le médiopassif parmi les structures à renversement}

Comme nous l'avons vu en 2.1., le médiopassif se caractérise par l'emploi du SN initialement complément du verbe en position sujet. De ce fait, il entre dans les structures 'à renversement' (Riegel, Pellat, Rioul 1994), dans lesquelles la position sujet de surface est occupée par un syntagme généré dans une autre position. Dans cette section, nous le comparons aux autres structures du même type.

\subsection{Comparaison avec les anticausatifs}

Une première analyse contrastive entre médiopassif et anticausatifs réfléchis a été proposée par Zribi-Hertz (1982). Ces deux structures se caractérisent en effet, en plus de l'alternance 
du sujet, par la présence d'un réfléchiiii ${ }^{\mathrm{iii}}$, comme le montre la schématisation (16), similaire à (2). Elles sont donc formellement identiques, ce que montrent les exemples (17-19).

16. a. SN1 V SN2

b. SN2 se-V

17. a. Le vent a cassé les branches

b. Les branches se sont cassées

18. a. La secousse a descellé le piton

b. Le piton s'est descellé

19. a. Le courant d'air a ouvert la porte

b. La porte s'est ouverte

Ce parallélisme confirme l'observation, faite par plusieurs auteurs sur diverses langues (voir par exemple Fábregas \& Putnam 2014 et les références citées), que le médiopassif n'a pas de structure propre. Au contraire, on observe à travers les langues qu'il est toujours formellement identique à d'autres constructions.

Les anticausatives se distinguent toutefois du médiopassif par le fait qu'elles admettent d'être associées aux expressions tout seul / de lui-même et sous l'effet de X (Zribi-Hertz 1982). A l'inverse, le médiopassif refuse toute expression syntaxique d'un syntagme renvoyant au sujet de la construction transitive correspondante. Ce contraste est illustré en $(20-21)$ :

20. Anticausatifs

a. Les branches se sont cassées \{toutes seules / d'elles-mêmes / sous l'effet du vent\}

b. Le piton s'est descellé \{tout seul / de lui-même / sous l'effet des secousses\}

c. La porte s'est ouverte \{toute seule / d'elle-même / sous l'effet du courant d'air\}

21. Médiopassif

a. *Ce sport se pratique \{tout seul / de lui-même / sous l'effet des compétitions\}

b. *Les feuilles mortes se ramassent \{toutes seules / d'elles-mêmes / sous l'effet du râteau\}

c. *La cathédrale se voit de loin \{toute seule / d'elle-même / sous l'effet de la lumière\}

d. *La choucroute se mange en hiver \{toute seule / d'elle-même / sous l'effet de la faim\}

Les exemples (16-18) ci-dessus se distinguent également des médiopassifs par le fait que les sujets transitifs des exemples (a) ne renvoient pas à des humains. Ils sont au contraire analysables comme des Causes (i.e. des entités non animées susceptibles de provoquer un événement), et non comme des Agents. Même si ce type de sujet est possible à la forme transitive, l'insertion dans des exemples tels que (20) d'un constituant renvoyant à un humain est agrammaticale, quelle que soit la préposition employée pour l'introduire.

22. a. *Les branches se sont cassées \{sous l'effet de Max / par Max\}

b. Max a cassé les branches

Les sujets originels des anticausatifs et des médiopassifs ne sont donc pas de même nature.

Enfin, les exemples ci-dessus nous permettent d'observer que la valeur épisodique n'est pas problématique pour les anticausatifs. 


\subsection{Comparaison avec le passif}

Comme le médiopassif, le passif se caractérise par le fait que le sujet de surface est initialement un complément à la forme active. Cependant, outre l'absence de pronom réfléchi, le passif admet l'expression du sujet transitif, quel que soit son rôle sémantique (23), sous forme d'un 'complément d'agent' introduit par par (Ruwet 1972, Lamiroy 1993).

23. a. Max a été embrassé par Marie Agent $_{\text {and }}$

b. Max est admiré par ses amis Experiencer

c. La maison a été détruite par le tremblement de terre Cause

Les exemples (24) et (25) illustrent le contraste entre passif et médiopassif.

\section{Passif}

a. Ce sport \{est / a été\} pratiqué par des sportifs de haut niveau

b. Les feuilles mortes \{sont / ont été\} ramassées par le jardinier

c. Les prises de sang \{sont / ont été\} faites par les infirmiers

\section{Médiopassif}

a. ${ }^{*}$ Ce sport se pratique par des sportifs de haut niveau

b. *Les feuilles mortes se ramassent par le jardinier

c. *Les prises de sang se font par les infirmiers

Observons en outre que les exemples (24) peuvent être interprétés comme épisodiques

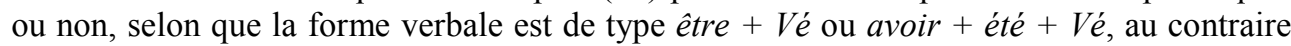
du médiopassif. Il n'existe donc pas de contrainte sur la valeur aspectuelle de la forme verbale passive.

\subsection{Comparaison avec les sujets instrumentaux}

Il nous semble également intéressant de comparer les phrases médiopassives à un troisième type de structure qui, comme les deux précédentes, a pour effet la démotion du sujet transitif. Il s'agit des phrases à sujets instrumentaux (Alexiadou \& Schäfer 2006, DeLancey 1994), présentées en (26).

26. a. Cette clé ouvre la porte (du garage)

b. Ce stylo (n')écrit (pas)

Ces structures présentent deux points communs avec le médiopassif. D'abord, elles ne peuvent pas comporter de SP renvoyant à un Agent ou à une Cause (27).

27. a. *Cette clé ouvre la porte \{par Max / sous l'effet de Max / sous l'effet du vent\}

b. *Ce stylo écrit \{par Max / sous l'effet de Max / sous l'effet de l'encre\}

Ensuite, elles sont difficilement compatibles avec l'interprétation épisodique. En d'autres termes, elles ne peuvent décrire un événement en cours, comme le montre l'impossibilité d'employer le progressif à la place du présent simple (28). Elles présentent au contraire des interprétations modales, du type de (29).

28. a. ??Cette clé est en train d'ouvrir la porte

b. ??Ce stylo est en train d'écrire 
29. a. Cette clé peut ouvrir la porte

b. Ce stylo (ne) peut (pas) écrire

Les phrases à sujet instrumental se distinguent cependant des autres structures présentées ci-dessus de deux manières. D'une part, elles ne présentent pas de marquage spécifique ; en d'autres termes, il n'existe pas de forme verbale instrumentale, cette valeur étant simplement produite par l'emploi d'un Instrument, par opposition à un Agent ou à une Cause, en position sujet :

30. a. [Marie $]_{\text {Agent }} /[\text { le courant d'air }]_{\text {Cause }}[\text { ouvre la porte du garage }]_{\mathrm{SV}}$

b. [Cette clé $]_{\text {Instrument }}[\text { ouvre la porte du garage }]_{\mathrm{SV}}$

D'autre part, les phrases instrumentales ne sont pas des structures à renversement. Comme le montre l'exemple (30), les SV d'une phrase à sujet instrumental et à sujet agentif / causal sont identiques.

Les propriétés du médiopassif et des autres constructions auxquelles nous venons de le comparer sont résumées dans le tableau 1.

Tableau 1 : Le médiopassif parmi les constructions à renversement

\begin{tabular}{|l|l|l|l|}
\hline $\begin{array}{l}\text { Transitif: } \\
\text { SN1 V SN2 (avec SN3) }\end{array}$ & Alternance & $\begin{array}{l}\text { Valeur } \\
\text { épisodique }\end{array}$ & $\begin{array}{l}\text { Expression du sujet } \\
\text { actif }\end{array}$ \\
\hline Médiopassif & SN2 se-V & non & impossible \\
\hline Anticausatif & SN2 se-V & oui & sous l'effet de SN1 \\
\hline Passif & SN2 être Vé & oui & par SN1 \\
\hline Sujets instrumentaux & SN3 $_{\text {Instr V SN2 }}$ & non & impossible \\
\hline
\end{tabular}

Ce tableau indique que, comme les phrases à sujets instrumentaux, les phrases médiopassives refusent l'expression d'une cause ou d'un agent, ainsi que la valeur épisodique. Il montre également que le médiopassif résulte de la combinaison de facteurs syntaxiques et sémantiques qui sont par ailleurs partagés par d'autres structures. Sur le plan syntaxique, il s'agit d'une structure réfléchie (comme les anticausatives) et dans laquelle la position sujet est occupée par le SN initialement complément du verbe (comme les anticausatives et le passif) ; sur le plan sémantique, elle se caractérise par la valeur nonépisodique.

\section{Valeurs du médiopassif}

Nous venons de voir que les constructions médiopassives ne permettent pas de dénoter un événement particulier, localisable dans le temps et/ou l'espace. De ce fait, comme elles refusent le progressif (9), elles ne constituent pas non plus des réponses appropriées à des questions de type Que se passe-t-il ? (Zribi-Hertz 2008) :

31. a. Que se passe-t-il ?

b. \# Les champignons se ramassent en forêt / \# la choucroute se mange en hiver / \# ce pull se lave en machine / ...

On peut ainsi rendre compte de l'inacceptabilité des exemples (32a, 33a), qui 
comportent un ancrage temporel exprimé par la référence à un moment précis :

32. a. * La poubelle s'est sortie hier soir

vs. b. La poubelle a été sortie hier soir

vs c. La poubelle se sort chaque soir

33. a. *Les vêtements se sont lavés en machine ce matin

vs. b. Les vêtements ont été lavés en machine ce matin

L'exemple (32c), montre que le remplacement d'une expression temporelle ancrée par une expression habituelle (chaque soir) restaure l'acceptabilité de la structure. En outre, pour renvoyer à un événement particulier, seul le passif sera utilisé (32b, 33b).

En l'absence de lecture épisodique, les phrases médiopassives prennent plusieurs valeurs. Melis (1990) repère des valeurs génériques ou descriptives (34a), Lagae (2002) observe également des lectures habituelles (34b). Nous pouvons ajouter des valeurs modales (34c,d), ou dispositionnelles (Lekakou 2005), dont certaines sont proches de celles des phrases à sujets instrumentaux (cf. (26)).

34. a. (Normalement / généralement) les champignons se ramassent en automne

b. La poubelle se sort chaque soir

c. Ce pull ne se lave pas à la machine

$=\quad c^{\prime}$. Ce pull ne $\{$ doit/ peut $\}$ pas se laver à la machine

d. La cathédrale se voit de loin

$=\quad d^{\prime}$. La cathédrale peut se voir de loin

Ces lectures relèvent de la catégorie aspectuelle que Smith (1991) nomme 'statifs dérivés'. Il s'agit de structures comportant des SV dynamiques (voir section 5.2.) mais qui fonctionnent comme des phrases statives, du fait qu'elles ne renvoient pas à un événement particulier. Plus spécifiquement, nous proposons, à la suite de Lekakou (2005), de décrire les phrases médiopassives comme des prédicats de propriétés, et non comme des prédicats événementiels, au sens où elles permettent l'expression de propriétés transitoires ou permanentes de leurs sujets. Ainsi, on peut observer que les phrases médiopassives peuvent répondre à la question Que sais-tu de $X$ ?, où $\mathrm{X}$ représente le sujet (cf. également ZribiHertz 2008), ce qui est également le cas des phrases à sujet instrumental (36) :

35. a. Que sais-tu \{des champignons / de la choucroute / de ce pull\}?

b. Les champignons se ramassent en forêt / la choucroute se mange en hiver / ce pull se lave en machine / ...

36. a. Que sais-tu de cette clé ?

b. Elle ouvre la porte du garage

Cette interprétation nous permet également de rendre compte du fait que certains verbes au médiopassif alternent librement avec les adjectifs en -able correspondants, illustrant ainsi la valeur dispositionnelle :

37. a. La laine se lave en machine

$=\quad$ b. La laine est lavable en machine

38. a. La cathédrale se voit de loin

$=\quad$ b. La cathédrale est visible de loin

39. c. Le bruit des vagues s'entend depuis la maison.

$=\quad \mathrm{c}$. Le bruit des vagues est audible depuis la maison. 
Cette alternance s'explique si l'on considère que de tels adjectifs caractérisent la propriété d'une entité vis-à-vis d'un type d'événement, et non son implication dans un événement particulier. Ainsi, au contraire d'un pull lavé, un pull lavable n'implique pas qu'un lavage ait eu lieu.

Pour conclure, nous considérons que les phrases médiopassives, au contraire de leurs variantes transitives, se caractérisent par l'émergence de valeurs modales ou habituelles, en l'absence de renvoi à un événement spécifique. De ce fait, elles constituent des prédicats non-spécifiants au sens de Kleiber (1981).

\section{Médiopassif vs passif : quels emplois ? quelles oppositions?}

\subsection{Alternance passif / médiopassif}

Les exemples (32-33) ci-dessus ont mis en évidence une opposition entre passif et épisodicité d'une part et médiopassif et non-épisodicité d'autre part. Cependant, Lagae (2002) observe dans certains cas une équivalence de sens entre les deux constructions, comme le montre (40), que nous complétons par (41-42).

40. a. Ce sport se pratique en plein air

$=\quad$ b. Ce sport est pratiqué en plein air

41. a. Le sorgho se cultive sur tous les continents

$=\quad$ b. Le sorgho est cultivé sur tous les continents ${ }^{\text {iv }}$

42. a. La linguistique s'enseigne à l'université

$=\quad \mathrm{b}$. La linguistique est enseignée à l'université

Dans la mesure où cette alternance semble marginale, on peut s'interroger sur ses conditions d'acceptabilité. Il s'avère que, du fait de la présence d'un verbe atélique, les structures (b) ci-dessus décrivent des situations en cours, au même titre que leurs équivalents médiopassifs. Ainsi, un sport pratiqué est un sport qu'on pratique actuellement, une plante cultivée est actuellement en culture, et une discipline enseignée l'est actuellement. Ces phrases ont donc une valeur imperfective ; ainsi, elles peuvent être employées à l'imparfait associées à une seconde phrase présentant la situation comme toujours en cours (Smith 1991).

43. a. Au $\mathrm{XIX}^{\circ}$ siècle, ce sport \{se pratiquait / était pratiqué\} en plein air. C'est encore le cas aujourd'hui.

b. Le sorgho \{se cultivait / était cultivé\} sur tous les continents, et il l'est toujours.

A l'inverse, dans les exemples (44-46), le médiopassif n'alterne pas avec le passif car les phrases passives décrivent non pas des événements en cours, mais des états résultants, c'est-à-dire des situations statives qui résultent de la réalisation d'un événement. Ainsi, des champignons ramassés ne sont pas des champignons qu'on ramasse actuellement, mais qui ont été déjà été cueillis. Du fait de ce caractère non-événementiel, elles ne peuvent être associées des expressions de lieu ou de manière (Maienborn 2003).

44. a. La cathédrale se voit de loin

$\neq \quad$ b. La cathédrale est vue ${ }^{\mathrm{v}}$ (?? de loin)

45. a. Les champignons se ramassent en forêt

$\neq \quad$ b. Les champignons sont ramassés (?? en forêt)

46. a. La laine se lave en machine

b. La laine est lavée (?? en machine) 
Ceci est confirmé par l'impossibilité d'employer encore ou toujours, qui marquent la reversibilité d'un état (Kratzer 2000) :

47. a. ?? La cathédrale est encore vue

b. ?? Les champignons sont encore ramassés (en forêt)

c. ?? La laine est encore lavée (en machine)

Nous concluons donc que si les phrases passives (40b-42b) alternent avec leurs équivalents médiopassifs en (a), c'est parce que ces deux structures présentent la propriété commune d'être imperfectives, au contraire des phrases passives (b) de (44-46). En d'autres termes, leur similarité interprétative découle de l'aspect lexical du verbe.

Notre observation rejoint ainsi celle d'Obenauer (1970) et de Ruwet (1972) à propos de la valeur imperfective du médiopassif, qu'ils relient au caractère non-épisodique de ces structures.

\subsection{Impossibilité du médiopassif}

Au delà des exemples présentés ci-dessus, la complémentarité du passif et du médiopassif est également interrogée par les paires d'exemples en (48-50).

48. a. Tout le monde aime les bonnes choses

b. Les bonnes choses sont aimées de tous

c. *Les bonnes choses s'aiment facilement

49. a. Beaucoup d'enfants \{adorent / détestent\} les épinards

b. Les épinards sont \{adorés / détestés\} par beaucoup d'enfants

b. *Les épinards \{s'adorent / se détestent\}

50. a. Cette famille \{habite / occupe / possède $\}$ ce château depuis plusieurs générations

b. Ce château est \{habité / occupé / possédé\} par la même famille depuis des générations

c. ${ }^{*}$ Ce château $\left\{\mathrm{s}^{\prime}\right.$ habite / s'occupe / se possède $\}$ depuis des générations

Au même titre que pratiquer, cultiver et enseigner (40-42), les verbes aimer, adorer, détester, habiter, occuper, posséder présentés dans les exemples ci-dessus sont atéliques. Employés au passif, ils décrivent des situations en cours, comme le montre (51). Toutefois, au contraire des premiers, les seconds n'admettent pas le médiopassif (cf. (48c-50c)).

51. a. Autrefois, les épinards étaient \{aimés /adorés / détestés\} par beaucoup d'enfants. C'est encore le cas aujourd'hui.

b. En 1950, le château était \{habité / occupé / possédé\} par la même famille depuis 7 générations et il l'est toujours.

Ils contrastent en cela avec les verbes voir (6), apercevoir ou entendre, également statifs :

52. a. Le bruit des vagues s'entend depuis la maison

b. Ce paysage montagneux s'aperçoit depuis le hameau de Pont Valsavarenche ${ }^{\mathrm{vi}}$

Or, il se trouve que les verbes qui n'admettent pas le médiopassif ne sont pas 
compatibles non plus avec la manière ou la localisation spatiale (53).

53. a. Beaucoup d'enfants \{aiment / adorent / détestent\} les épinards $\{*$ calmement $/ *$ au restaurant $\}$

b. Cette famille possède le château $\left\{{ }^{*}\right.$ tranquillement / *à la campagne $\left.{ }^{\mathrm{vii}}\right\}$

Selon Maienborn (2005), cette restriction permet d'analyser les verbes aimer, adorer, détester, posséder, occuper et habiter comme de véritables statifs (ou 'états kimiens'), au contraire de voir, apercevoir ou entendre, qui sont des 'états davidsoniens', et comportent une composante événementielle. L'absence de composante événementielle à l'actif explique ainsi la proximité sémantique avec le passif (cf. (48-50a vs b), aucun des deux n'étant événementiel. De ce fait, dans de tels exemples, encore / toujours signalent que les situations statives sont en cours (54). Comme le médiopassif, les formes en être + Vé sont donc imperfectives.

54. a. Les épinards sont \{encore / toujours\} \{adorés / détestés $\}$ par les enfants

b. Ce château est \{encore / toujours\} \{habité / occupé / possédé\} par la même famille

$\mathrm{Au}$ vu du contraste entre ces deux classes de verbes, nous pouvons conclure que, pour un verbe donné, la présence d'une composante événementielle est un préalable à l'emploi du médiopassif.

\section{Médiopassif et perfectivité}

Dans ce qui précède, nous avons vu que le médiopassif est une forme verbale imperfective. Cependant, Obenauer (1970) et Ruwet (1972) considèrent que certaines structures médiopassives admettent l'emploi perfectif. Melis (1990) considère même que les deux aspects sont toujours disponibles. Le médiopassif présenterait alors une interprétation épisodique et renverrait à un événement ponctuel (Lagae 2002, Zribi-Hetz 2008). Ceci est illustré en (55-56). Notons que le SP temporel de l'exemple (56a) permet de présenter l'événement comme en cours, ce qui contraste avec la valeur habituelle ou modale du présent dans les exemples observés plus haut.

55. a. La décision s'est prise hier

b. L'usine s'est construite en quelques semaines

c. L'appartement s'est loué en 28 jours seulement

d. Les Tournesols s'est vendu 40 millions d'euros

56. a. Le match se joue en ce moment même

b. La finale se disputera à partir de lundi

c. La bataille de Bouvines s'est livrée le 27 juillet 1214

Au vu de ce qui précède, et notamment d'exemples tels que (32-33), complétés par (57), la question se pose de savoir si les exemples ci-dessus sont réellement des médiopassifs, et s'il ne s'agit pas plutôt d'anticausatifs.

57. a. ?? L'Amérique s'est découverte en 1492

b. ?? La poubelle s'est sortie hier soir

c. ?? Ces lunettes se sont nettoyées hier matin

L'hypothèse anticausative se voit confirmée pour les exemples (55) par le fait qu'ils 
admettent d'être associés à des syntagmes introduits par tout(e) seul(e), sous l'effet de, sous la pression de ou encore sous l'influence de, qui expriment la Cause :

58. a. Sous la pression des pouvoirs publics, l'usine s'est construite en quelques semaines (seulement)

b. Sous l'effet de la pénurie de logements, l'appartement s'est loué en 28 jours seulement

c. \{Sous l'effet de la publicité / sous la pression d'un enchérisseur anonyme\}, Les Tournesols s'est vendu 40 millions d'euros

d. La décision s'est prise toute seule ${ }^{\text {viii }}$

Les exemples (56) sont cependant différents. D'une part, on peut remarquer que l'expression d'une Cause et tout seul sont exclus :

59. a. Le match se joue en ce moment même $\{*$ tout seul / ?? sous l'influence du public\}

b. La finale se disputera à partir de lundi \{*toute seule / ?? sous l'effet de la météo\}

c. La bataille de Bouvines s'est livrée le 27 juillet 1214 \{*toute seule / ?? sous la pression du roi de France\}

Ensuite, on observe que les sujets renvoient à des événements, les verbes médiopassifs pouvant alterner avec avoir lieu et se dérouler (Godard \& Jayez 1994):

60. a. Le match $\{$ a lieu / se déroule $\}$ en ce moment même

d. La finale \{aura lieu / se déroulera $\}$ à partir de lundi

e. La bataille de Bouvines \{a eu lieu / s'est déroulée\} le 27 juillet 1214

La présence de en ce moment même (56a), à partir de lundi (b) et le 27 juillet 1214 (c) atteste du fait que les événements décrits par les sujets sont ancrés dans le temps, donc spécifiques. De ce fait, dans de telles structures, la lecture non-épisodique est impossible ; ainsi, les exemples (61) sont mal formés, sauf à interpréter les sujets comme des événements récurrents chaque semaine $\left(a^{\prime}, b^{\prime}\right)$ ou chaque année $\left(c^{\prime}\right)$ :

61. a. ? Le match se joue le mardi

$=\quad a^{\prime}$. Les matchs se jouent (toujours) le mardi

b. ? La finale se dispute le lundi

$=\quad b^{\prime}$. Les finales se disputent chaque lundi

c. ? La bataille se livre le 27 juillet

$=\quad c^{\prime}$. Les batailles se livrent le 27 juillet (de chaque année)

Nous supposons donc que la disponibilité de la lecture épisodique d'exemples tels que (56) découle du caractère spécifique de l'événement décrit par le sujet, puisqu'en l'absence d'un sujet de ce type (cf. (57)), elle n'est pas disponible. La lecture épisodique n'est donc pas une propriété du médiopassif.

Observons pour finir que c'est bien le caractère non-épisodique du médiopassif qui provoque l'inacceptabilité du perfectif, et non pas la forme verbale composée en elle-même. Ainsi, les phrases médiopassives au passé composé sont parfaitement acceptables lorsqu'elles décrivent des événements passés habituels (voir Kleiber 1987 et Boneh \& Doron 2010) : 
62. a. Ca s'est beaucoup dit / fait à une certaine époque

b. La phrénologie s'est enseignée à l'université au XIX ${ }^{\circ}$ siècle

c. Pendant très longtemps, les anneaux de Saturne ne se sont pas vus.

d. En réalité, ce disque s'est vendu "sous le manteau" à la sortie de l'église et n'a été tiré qu'à 500 exemplaires ${ }^{\mathrm{ix}}$.

De tels exemples confirment que le médiopassif est bien non-épisodique. Lorsque ce n'est pas le cas, l'épisodicité est due à des facteurs qui ne relèvent pas de la forme verbale.

\section{Bilan}

Dans ce travail, nous avons examiné les propriétés du médiopassif, à partir d'observations antérieures de plusieurs auteurs. Sur le plan de l'aspect lexical, nous avons montré que les structures médiopassives sont nécessairement construites à partir de verbes comportant une composante événementielle, les verbes purement statifs (ou états kimiens) en étant exclus. Sur le plan argumental, elles se caractérisent par l'élimination de l'argument externe de la structure transitive, qui renvoie prioritairement à un humain. Contrairement à ce qui se produit au passif et avec les verbes anticausatifs réfléchis, cet argument ne peut pas être restitué sous la forme d'un SP. Cette propriété est partagée par les structures à sujet instrumental, de même que la lecture non-épisodique, ce qui laisse supposer qu'il existe une relation entre ces deux phénomènes. En l'absence de renvoi à un événement particulier, les phrases médiopassives présentent différents types de valeurs, notamment habituelles et modales, et s'interprètent comme décrivant une propriété de leur sujet, au même titre que les phrases à sujet instrumental. Nous avons également montré que le médiopassif est une forme verbale intrinsèquement imperfective, et que c'est cette propriété qui rend compte de ses relations d'équivalence ou d'opposition avec les formes passives (être +Vé).

\section{Références}

Alexiadou, A. \& Schäfer, F. (2006). Instruments subjects are Agents or Causers. In D. Baumer, D. Montero, and M. Scanlon (eds). Proceedings of the 25th West Coast Conference on Formal Linguistics. Somerville : MA: Cascadilla Proceedings Project, 40-48.

Boneh, N. \& Doron E. (2010). Modal and Temporal Aspects of Habituality. In M. Rappaport-Hovav, E. Doron and I. Sichel (eds.) Syntax, Lexical Semantics, and Event Structure. Oxford : Oxford University Press.

DeLancey, S. (1984). Notes on agentivity and causation. Studies in Language, 8-2, 181-213.

Fábregas, A. \& Putnam, M. (2014). The emergence of middle voice structures with and without agents. The Linguistic Review 31-2, 193-240.

Godard, D. \& Jayez, J. (1994). Types nominaux et anaphores : le cas des objets et des événements. Cahiers Chronos 1, 41-58.

Kemmer, S. (1993). The middle voice. Amsterdam : John Benjamins

Kleiber, G. (1981). Problèmes de référence : descriptions définies et noms propres. Paris : Klincksieck.

Kleiber, G. (1981). Du côté de la référence verbale : les phrases habituelles. Berne : Peter Lang.

Kratzer, A. (2000). Building statives. Berkeley Linguistic Society, 26, 385-399.

Lagae V. (2002). Le passif pronominal : une forme complémentaire du passif périphrastique ? In V. Lagae, A. Carlier, C. Benninger (eds) Temps et aspect: de la grammaire au lexique. Amsterdam : Rodopi. 133-149.

Lamiroy B. (1993). Pourquoi il y a deux passifs. Langages, 109, 53-72.

Lekakou, M. (2005). In the middle, somewhat elevated. The semantics of middles and its crosslinguistic realization. Ph.D. Dissertation. University College, London.

Maienborn, C. (2005). On the limits of the Davidsonian approach: The case of copula sentences. Theoretical linguistics, 31-3, 275-316. 
Melis, L. (1990). La voie pronominale. Paris / Louvain-la-Neuve : Duculot.

Obenauer, H. (1970). La construction pronominale passive en français moderne. Mémoire de maîtrise, Université Paris 8.

Reinhart, T. \& Siloni, T. (2005). The Lexicon-Syntax Parameter: reflexivization and other arity operations. Linguistic Inquiry 36-3, 389-436.

Riegel M., Pellat, J.C. \& Rioul, R. (1994). Grammaire méthodique du français. Paris : Presses Universitaires de France.

Ruwet, N. (1972). Théorie syntaxique et syntaxe du français. Paris : Seuil.

Smith, C.S. (1991). The Parameter of Aspect. Dordrecht : Kluwer

Zibi-Hertz, A. (1982). La construction 'se-moyen' du français et son statut dans le triangle : moyenpassif-réfléchi, Lingvisticae Investigationes 6, 345-401.

Zibi-Hertz, A. (2008). Le médiopassif à accord riche en français : une analyse multifactorielle. Actes du CMLF, https://doi.org/10.1051/cmlf08083.

\footnotetext{
${ }^{\mathrm{i}}$ http://www.lesdebrouillards.com/quoi-de-neuf/ebola-ce-quil-faut-savoir/

ii members.tripod.com/ SPA_Laberge/diete.htm

iii Le français présente également des anticausatives non-réfléchies, cf. le pâtissier a brulé le gâteau / le gâteau a brulé.

${ }^{i v}$ solidaritefsm2011.blogspot.com/2014/.../pour-quand-le-grand-retour-du-sorgho-23

${ }^{v}$ On constate que voir s'interprète comme 'devenir visible', et prend ici la valeur d'un achèvement.

${ }^{v i}$ https://www.pinterest.fr/pin/436919601325628423/?autologin=true

${ }^{v i i}$ Cet exemple est bien entendu acceptable si le SP locatif est interprété comme une dépendance du nom château, cf. [le château [à la campagne $\left.]_{\mathrm{SP}}\right]_{\mathrm{SN}}$

viii lachalouperassemble.over-blog.com/article-28390908.html

${ }^{\text {ix }}$ www.priceminister.com > Disque vinyle > Variété française
} 